\title{
Elaboração, Análise e Aplicação de um Modelo Epistemológico de Referência para o Ensino do Conceito de Função na Licenciatura em Matemática
}

\author{
Elaboration, Analysis, and Application of a Reference Epistemological \\ Model for Teaching the Function Concept on a Mathematics Degree Course
}

Rochelande Felipe Rodrigues*

ORCID iD 0000-0003-4439-6425

Catarina Oliveira Lucas ${ }^{* *}$

ORCID iD 0000-0002-0645-2169

São Luís Castro***

ORCID iD 0000-0002-1487-3596

Marcus Bessa de Menezes ${ }^{* * * *}$

ORCID iD 0000-0003-0850-1793

Marcelo Câmara dos Santos ${ }^{* * * * *}$

ORCID iD 0000-0002-6466-9040

\begin{abstract}
Resumo
O artigo trata da elaboração, análise e aplicação do Modelo Epistemológico de Referência (MER) para o conceito de função que foi aplicado na disciplina de Funções I da licenciatura em Matemática da Educação do Campo. Para a sua elaboração foram utilizadas algumas informações preliminares sobre o conceito de função, tais como: uma breve evolução histórica do conceito; algumas dificuldades no processo de ensino e aprendizagem; a sua organização nos documentos curriculares brasileiros; e uma análise praxeológica de um livro didático utilizado na disciplina pesquisada. Como base teórica da elaboração do nosso MER utilizamos: a proposta de Lucas aplicada ao cálculo diferencial elementar; a modelação matemática de Chevallard e Gascón; e os níveis de modelação funcional de Ruiz-Munzón. Com essa organização, propomos um esquema de um MER para o conceito de função, que fundamentou a aplicação do Percurso de Estudo e Pesquisa (PEP), aplicado pelo professor da disciplina de

\footnotetext{
* Doutor em Ensino de Ciências e Matemática pela Universidade Federal Rural de Pernambuco (UFRPE). Professor do Instituto de Formação de Educadores da Universidade Federal do Cariri (UFCA), Brejo Santo, Ceará, Brasil. Email: felipemtm@gmail.com.

** Doutorada pela Universidad de Vigo (UVigo). Professora no Instituto Superior Politécnico Gaya (ISPGAYA), Vila Nova de Gaia, Porto, Portugal. E-mail: clucas@ispgaya.pt.

*** Doutorada em Psicologia Experimental pela Universidade do Porto (UP). Professora catedrática na Faculdade de Psicologia e Ciências da Educação da Universidade do Porto (FPCEUP), Porto, Portugal. E-mail: slcastro@fpce.up.pt.

***** Doutor em Educação pela Universidade Federal de Pernambuco (UFPE). Professor Associado da Universidade Federal de Campina Grande (UFCG), Sumé, Paraíba, Brasil. E-mail: marcusbessa@gmail.com

****** Doutor em Ciências da Educação pela Université Paris-X. Professor do Programa de Pós Graduação em Educação Matemática e Tecnológica da Universidade Federal de Pernambuco (EDUMATEC), Recife, Pernambuco, Brasil. E-mail: marcelocamaraufpe@yahoo.com.br.
} 
Funções I para seus alunos no período de seis sessões. Durante o processo de aplicação, foi observado o desenvolvimento do PEP fundamentado pelo MER, que se apresenta como possibilidade para o ensino de função, destacando o processo de transição de um domínio discreto para o contínuo, como também, o estudo de famílias de funções. Os resultados encontrados indicam que as técnicas não tomam um papel de destaque em relação à compreensão dos conceitos, ocasionando uma mudança da postura do professor para proporcionar o ensino e aprendizagem do conceito de função.

Palavras-chave: Teoria Antropológica do Didático; Modelo Epistemológico de Referência; Função.

\begin{abstract}
This article deals with the elaboration, analysis, and application of the reference epistemological model (REM) for the function concept, that was applied in the Functions I discipline of the Field Education Mathematics degree. For model elaboration, we used preliminary information about the function concept, as: a brief historical evolution of the concept; some difficulties in the teaching and learning processes; its organization in the Brazilian curriculum documents; and a praxeological analysis of a textbook used in the researched subject. As a theoretical basis for the elaboration of our REM, we used: a proposal by Lucas applied to the concept of elementary differential calculus; the mathematical modelling notion of Chevallard and Gascón and the functional modelling levels proposed by Ruiz-Munzón. With this organization, we proposed a schema of a REM for the function concept, that fundamented the application of Study and Research Path (SRP), which was applied by the teacher of the Functions I discipline to his students over a period of six sessions. During the application process, we were able to observe the development of the SRP fundamented by REM, which presents itself as a possibility for teaching the function concept. We highlight the transition process from discrete to continuous domain, as well as the study of families of functions. The results showed that the techniques do not take a prominent role in relation to the understanding of concepts, causing a change in the teacher's perspective on how to teach and facilitate learning of the function concept.
\end{abstract}

Keywords: Anthropological Theory of the Didactic. Reference Epistemological Model. Function.

\title{
1 Introdução
}

O professor, ao iniciar o processo de ensino dos conceitos matemáticos, conduz a sua prática docente de modo a possibilitar uma transformação no saber em jogo, buscando meios que proporcionem, na maioria dos seus alunos, a compreensão e aplicação dos conceitos ensinados. A condução da prática docente geralmente está atrelada a alguma proposta de ensino, em que diferentes caminhos metodológicos utilizados pelos professores podem auxiliar no processo de ensino e aprendizagem dos conceitos matemáticos.

As pesquisas das propostas no ensino da Matemática proporcionam uma visão dos possíveis caminhos que podem ser utilizados para buscar uma diminuição dos problemas relacionados à compreensão dos conceitos. Um dos problemas está ligado à compreensão do conceito de função, no qual alguns alunos não conseguem relacionar a dependência entre as grandezas envolvidas e suas diferentes formas de representação (LOURENÇO; OLIVEIRA, 2014; TENÓRIO; PENA; TENÓRIO, 2015; ANDRADE; SARAIVA, 2012).

Este artigo está relacionado às dificuldades em torno da compreensão do conceito de função e apresentará resultados parciais de uma pesquisa de doutoramento. Ao longo do texto, 
iremos destacar a construção e a análise do modelo epistemológico de referência ${ }^{1}$ (MER) para o ensino de funções elementares, que representa uma parte dos resultados coletados e analisados durante o doutorado. A pesquisa iniciou com uma reflexão sobre as bases conceituais da Teoria Antropológica do Didático (TAD), especificamente na do Percurso de Estudo e Pesquisa ${ }^{2}$ (PEP), com elementos teóricos fundamentais para a compreensão do seu desenvolvimento (RODRIGUES; MENEZES; CAMÂRA DOS SANTOS, 2017). Este artigo discute alguns resultados gerados a partir do MER proposto, por meio do desenvolvimento da questão geratriz $\left(Q_{0}\right)$ de um PEP.

Dentre as teorias da Didática da Matemática, optamos pela Teoria Antropológica do Didático (TAD), que proporciona a compreensão do processo de ensino dos conceitos matemáticos e de sua aplicação, estabelecendo uma relação codisciplinar e interdisciplinar no processo de ensino.

A TAD propõe estudos que podem auxiliar na compreensão de alguns problemas existentes nas instituições de ensino. Os problemas podem ter diferentes dimensões, mas destacamos os seguintes: o monumentalismo escolar (CHEVALLARD, 2006), no qual o conhecimento é visitado de forma isolada e desarticulada; a fraude epistemológica (D'AMORE, 2007), em que os alunos respondem às atividades a partir de reprodução de resultados de atividades anteriores, acreditando que compreenderam o conceito estudado; e o autismo institucional (PARRA; OTERO, 2011), no sentido de que existe a falta de comunicação eficiente entre as universidades e a sociedade. Diante destes problemas, podemos concordar com o alerta de Onuchic e Allevato (2009), ao ressaltarem que os professores de Matemática estão saindo das universidades mal preparados para enfrentar os problemas de ensino existentes na Educação Básica.

A TAD tem sua fundamentação ancorada no paradigma do questionamento do mundo, para se contrapor ao paradigma do inventário dos saberes (CHEVALLARD, 2009a, 2009b), buscando proporcionar um ensino e uma aplicação não isolados dos conceitos matemáticos escolares, articulando seu entendimento e aplicações nas diferentes áreas da Matemática.

Segundo Chevallard (1996), a TAD deve ser observada como um desenvolvimento e articulação das noções praxeológicas que permitam pensar de forma unificada um considerável número de fenômenos didáticos. Segundo Bosch e Gascón (2010), a TAD apresenta as formas

\footnotetext{
1 O MER é um modelo cuja organização matemática segue uma organização didática e apresenta-se como alternativa frente à epistemologia dominante na instituição de ensino.

${ }^{2} \mathrm{O}$ PEP é aplicado e desenvolvido tomando por base a complexidade crescente das organizações matemáticas, proporcionando a construção de sequências de ensino e ocasionando uma mudança praxeológica.
} 
de organização do ensino escolar de Matemática e são descritas em termos de praxeologias de ensino com dois blocos em sua estrutura: prático-técnico (a práxis, o saber fazer didático) e o tecnológico-teórico (o logos, o saber didático). A práxis didática é constituída pelos tipos de tarefas $(\mathrm{T})$ e pelas técnicas $(\tau)$ que estão disponíveis quando há um discurso tecnológico $(\theta)$ teórico $(\Theta)$ didático capaz de descrever, desenvolver e explicar a prática didática, possibilitando a criação de critérios para aperfeiçoá-las (LUCAS, 2015). Chevallard (2009a) caracteriza um sistema didático $(S)$ como uma reunião de três instâncias: estudante $(x \in X)$, professor $(y \in Y)$ e desafio didático $(\bullet)$, no qual o estudante tenta resolver o desafio didático: $S(X, Y, \bullet)$.

O desafio didático pode ser desenvolvido tomando por base o Percurso de Estudo e Pesquisa (PEP). Almouloud e Silva (2012) comentam que o PEP é um tipo de engenharia didática (ARTIGUE, 1996) com algumas características próprias, denominada engenharia do $\mathrm{PER}^{3}$. Chevallard $(2004,2006,2009 a)$ apresenta o PEP como um dispositivo didático direcionado à modelação matemática ${ }^{4}$, relacionado ao estudo de uma questão geratriz $\left(Q_{0}\right)$, gerando outras questões derivadas $\left(Q_{i}\right)$. A $Q_{0}$ e suas derivadas $\left(Q_{i}\right)$ possibilitam respostas diferentes $\left(R_{i}\right)$, levando a uma maior articulação dos conceitos matemáticos, assim como das técnicas fundamentadas pelas tecnologias. Com isso, Chevallard (2009a) apresenta um modelo baseado no "esquema herbatiano"

$$
\left[S(X ; Y ; Q) \rightarrow\left\{R_{1}^{0}, R_{2}^{0}, \ldots, R_{n}^{0}, O_{n+1}^{0}, \ldots, O_{m}^{0}\right\}\right] \rightarrow R^{\vee}
$$

O modelo apresenta, durante o desenvolvimento do estudo, todos os recursos médios ${ }^{6}$, os saberes e as respostas disponíveis $R_{i}^{\diamond}$, para construir uma boa resposta $R^{\boldsymbol{}}$, na qual os objetos de qualquer natureza $O^{\natural}$ atuarão como médios para colocar em prova as $R_{i}^{\diamond}$.

Tomando por base a condução metodológica proposta pelo PEP, elaboramos em nossa pesquisa uma questão geratriz $\left(Q_{0}\right)$ que gerou outras questões derivadas $\left(Q_{1} e Q_{2}\right)$ e, consequentemente, derivadas das derivadas, aglomerando um conjunto de questões organizadas cognitivamente que mobilizam objetos $O^{\diamond}$ e respostas $R_{i}^{\diamond}$, a fim de buscar uma boa resposta $R^{\natural}$.

\footnotetext{
${ }^{3}$ Parcours d'Étude et de Recherche (PER) é a sigla em francês do Percurso de Estudo e Pesquisa (PEP). 4 A TAD descreve a modelação matemática como um processo de construção e reconstrução das Organizações Matemáticas complexas e crescentes (Organização Matemática Pontual, Organização Matemática Local, Organização Matemática Regional, Organização Matemática Global), partindo de uma questão problemática que constitui a razão de ser do processo (LUCAS, 2015).

${ }^{5}$ Segundo Lucas (2015, p. 33), o esquema herbartiano pode ser considerado "um sistema de referência didático para observar, descrever, analisar e avaliar os sistemas existentes nas instituições sociais ou teoricamente possíveis". Este esquema fornece um modelo geral entendido pela TAD como «estudar uma questão».

${ }^{6}$ Instrumento indispensável para colocar à prova as respostas geradas a partir das perguntas, verificando a sua validez (BOSCH; GASCÓN, 2010). É importante destacar que o sentido do médio vai além do apresentado no texto, o qual podemos associar ao termo "milieu" discutido por Brousseau.
} 
A aplicação do PEP em nossa pesquisa foi fundamentada pela proposta de um possível modelo epistemológico de referência (MER) para o ensino de funções elementares na licenciatura em Matemática, em particular, uma licenciatura direcionada à Educação do Campo $^{7}$. Os modelos epistemológicos de referência são hipóteses de trabalho, podendo ser, constantemente, questionados e revisados, o que possibilita a análise do saber matemático, antes de ser ensinado. O modelo pode proporcionar uma visão dos principais problemas de compreensão conceitual e apresentar possíveis caminhos para a solução desses problemas (GASCÓN, 2011). O MER apresenta-se como uma alternativa frente à epistemologia dominante na instituição de ensino. Gascón (2014) destaca que,

para ter os processos de transposição didática como objeto de estudo, a didática necessita analisar de maneira crítica os modelos epistemológicos das matemáticas dominantes nas instituições envolvidas, e assim libertar-se de ter de assumir esses modelos sem sentido crítico (GASCÓN, 2014, p. 100, tradução nossa) ${ }^{8}$.

Tal enfrentamento é materializado pelo MER, em que o estudo crítico dos documentos oficiais, das concepções dos professores e alunos, dos livros de texto, dos planos de estudos, das praxeologias atuais, entre outros, pode fazer parte de sua construção inicial. Destacamos que a proposta está passível de mudanças, ou adaptações, no decorrer das futuras aplicações de outros PEP, até porque os resultados apresentados neste artigo estão limitados ao conceito de função e de função afim, necessitando de mais aplicações, tomando por base outros PEP que abordem outros tipos de funções, tais como as quadráticas. Também existe a necessidade de ser aplicado em outras modalidades de licenciaturas em Matemática para possibilitar uma atuação ampla.

\section{Construção do Modelo Epistemológico de Referência para o ensino de função a ser utilizado na Licenciatura em Matemática: uma abordagem na Educação do Campo}

Para a construção do MER, consideramos alguns pontos preliminares sobre o conceito de função, tais como: alguns elementos históricos; a sua organização nos documentos oficiais educacionais brasileiros; as dificuldades e avanços do processo de ensino e aprendizagem, tomando por base algumas pesquisas; e elementos praxeológicos do livro de Matemática

\footnotetext{
${ }^{7}$ Segundo o Ministério da Educação e Cultura (MEC) do governo brasileiro, as licenciaturas em Educação do Campo têm o objetivo de "apoiar a formação inicial de professores em exercício na educação do campo e quilombola, assegurando condições de acesso aos cursos de licenciatura destinados à atuação docente nos anos finais do ensino fundamental e no ensino médio" (MEC, 2019).

8 "para tomar los procesos de transposición didáctica como objeto de estudio, el didacta necesita analizar de manera crítica los modelos epistemológicos de las matemáticas dominantes en las instituciones involucradas y liberarse así de la asunción acrítica de dichos modelos"(GASCÓN, 2014, p. 100).
} 
elementar, geralmente utilizado nas licenciaturas em Matemática. Estes pontos preliminares foram analisados e utilizados para construir uma proposta do Modelo Epistemológico de Referência (MER) que fundamentou a aplicação do Percurso de Estudo e Pesquisa.

Em relação aos elementos históricos, observamos que o conceito de função, no decorrer do tempo, sofreu mudanças e sua compreensão foi ficando mais ampla, à medida que as contribuições dos matemáticos na elaboração da definição de função foram sendo incorporadas; consequentemente, a sua aplicação também foi expandida para novos campos de estudos, a exemplo do cálculo diferencial e integral. As definições de função apresentadas no decorrer da sua história, até sua abordagem nos livros didáticos, trazem a sua evolução conceitual e sua aplicação na área da Matemática, assim como em outras áreas de conhecimento. Percebemos, na sua apresentação nos livros didáticos, que existiu a necessidade de deixar a definição de função mais compreensível para os alunos que estudam na Educação Básica, como também no Ensino Superior, com a intenção de permitir a compreensão de sua importância no campo da Matemática e em outras áreas de conhecimento (EVES, 2004; BOYER, 1996). É importante destacar que os elementos históricos abordados estão limitados ao conceito, que deverá ser ampliado, de acordo com o aprofundamento do MER proposto.

No caso dos documentos oficiais da Educação Brasileira, que têm como um dos seus objetivos orientar as conduções conceituais e didáticas das instituições de ensino, destacam-se os principais pontos considerados essenciais no processo de ensino e aprendizagem. Dentre esses documentos, podemos citar os Parâmetros Curriculares Nacionais do Ensino Médio (PCN) do terceiro e quarto ciclos do Ensino Fundamental, as Orientações Curriculares para o Ensino Médio, os pareceres do Conselho Nacional de Educação (CNE) e a recente Base Nacional Comum Curricular (BNCC). A análise desses documentos, deu-nos uma fundamentação para compreender as orientações curriculares do conteúdo de função na Educação Básica brasileira e sua influência nos cursos de licenciaturas em Matemática, o que ressaltou sua importância conceitual em Matemática, como também na aplicação em outras áreas de conhecimento (BRASIL, 1998, 2000, 2006, 2017).

Um ponto importante para a elaboração do MER foi a identificação e entendimento de alguns problemas que dificultam a compreensão e a aplicação do conceito de função, o que se tornou essencial para propormos outros caminhos de ensino alternativos que atuem na diminuição dos problemas em sala de aula. Observamos algumas pesquisas que abordaram temas como: a discussão superficial do conceito de função e a falta de conexão entre as suas representações (ESPITA; CRUZ; OCHOA, 2011); a dificuldade em relacionar os tipos diferentes de registros (LOURENÇO; OLIVEIRA, 2014); a falta de compreensão da 
dependência entre as variáveis (TENÓRIO; PENNA; TENÓRIO, 2015); a dificuldade em relacionar o conceito de função como fundamental e lógico-histórico, sem uma relação com os problemas do cotidiano (NEVES; RESENDE, 2016); e a dificuldade em compreender a funcionalidade dos símbolos matemáticos relacionados ao conceito de função (LIMA, 2014).

Complementando a discussão para a construção da proposta do Modelo Epistemológico de Referência (MER), realizamos uma análise das tarefas, técnicas, tecnologias e teorias presentes no livro didático Fundamentos da Matemática Elementar - volume 1 (IEZZI; MURAKAMI, 2004) que geralmente é utilizado em vários cursos de licenciatura em Matemática e está presente nas referências das ementas de disciplinas que abordam conteúdos dos fundamentos da Matemática. A análise dos elementos praxeológicos do conceito de função e de função afim, no livro didático, possibilitou observar alguns problemas encontrados: repetição excessiva de técnicas; apresentação superficial do conceito; falta de articulação entre os conceitos e suas representações; excesso de símbolos matemáticos sem uma discussão da sua funcionalidade; utilização inadequada de problemas e valorização da representação algébrica.

O estudo desses elementos preliminares para a construção do nosso MER foi essencial para identificar as práticas dominantes utilizadas no processo de ensino do conceito de função e de suas aplicações. Ressaltamos que essa foi uma análise preliminar para iniciarmos uma proposta de ensino do conceito de função para a licenciatura em Matemática.

\subsection{Formulação do problema didático para a elaboração do MER}

Com os elementos preliminares analisados, partimos para a formulação do problema didático, tomando por base três dimensões: a epistemológica, a econômica-institucional e a ecológica. A análise dessas dimensões nos deu uma orientação e nos conduziu à continuidade do desenvolvimento do MER, focando nos problemas do ensino do conceito de função. Para esquematizar o desenvolvimento do problema didático, utilizamos a seguinte representação (GASCÓN, 2011):

$$
\left\{\left[\left(P_{0} \oplus P_{1}\right) \subset P_{2}\right] \subset P_{3}\right\} \subset P_{\delta}
$$

Gascón (2011, p. 206) explica que $P_{1}, P_{2}$ e $P_{3}$ são as dimensões fundamentais do problema didático, e que o $P_{0}$ tem um papel especial, pelo fato de representar a formulação do problema inicial; tais problemas podem ter características “pré-científicas”, que geralmente são visíveis e presentes em todos os problemas didáticos. $\mathrm{O}$ símbolo $\oplus$ indica que o $P_{0}$ é incompleto, existindo a necessidade de ser adicionado à dimensão epistemológica $P_{1}$, para, pelo 
menos, ser considerado um problema. No caso de $\subset$, é entendido como inclusão, indicando que cada uma das dimensões $P_{i}$ é logicamente anterior a $P_{i+1}$, caracterizando um hipotético desenvolvimento do problema. Finalmente, o $P_{\delta}$ é denominado de problema didático, contendo as três dimensões fundamentais.

O problema $P_{0}$ tem seu ponto de partida quando o professor inicia um conteúdo matemático junto a seus alunos. Os problemas são formulados sob a influência da cultura escolar existente, podendo ser fundamentados nos documentos escolares, que, muitas vezes, não são questionados pelos professores. Nesse sentido, representaremos $P_{0}$ pela seguinte pergunta: $P_{0}(F)$ : Como ensinar aos alunos o conceito de função? Ao propor o problema inicial, outros problemas específicos também podem ser enunciados: $P^{\prime}{ }_{0}(F)$ : Como posso motivar os alunos a ampliarem o interesse em estudar o conceito de função? Como utilizar os recursos da Tecnologia da Informação e Comunicação (TIC) para o ensino de função? Como fazer com que cada aluno compreenda o conceito de função? Gascón (2011) destaca que todo problema didático se inicia por um problema construído pelo professor, no caso, por um problema docente.

A dimensão epistemológica $P_{1}$ está ancorada em um modelo epistemológico de referência (MER) que é provisório e, com base nele, pode-se analisar, construir ou reconstruir as praxeologias presentes na instituição a ser analisada. Gascón (2011) destaca que a dimensão epistemológica é um componente essencial na construção do problema didático, por tratar de questões epistemológicas do saber em jogo. Algumas perguntas relacionadas à dimensão epistemológica direcionada ao conceito de função podem ser formuladas: $P_{1}(F)$ : O que é função e qual a sua aplicação na Matemática? Como ensinar o conceito de função, tomando por base o MER? Como relacionar a dependência entre as variáveis de uma função? Como proporcionar a compreensão da mudança de registro de uma função? Como aplicar as definições de função crescente ou decrescente em qualquer tipo de função?

Gascón (2011) comenta que a dimensão econômica-institucional gira em torno de perguntas relacionadas às Organizações Matemáticas (OM) e às Organizações Didáticas (OD) existentes em uma determinada instituição, como também desenvolve, experimenta e analisa novas OD. Ao introduzir novas OD, existirá a possibilidade de análise dos fatos didáticos produzidos no sistema docente, fatos ocasionados por essas novas organizações, assim como a análise de sua viabilidade. No caso, temos as seguintes perguntas: $P_{2}(F)$ : Como é interpretado e compreendido o conceito de função nos cursos de Licenciatura em Matemática atualmente? Como se apresenta a rigidez e a desarticulação das OM, ao se abordar o conceito de função, nos 
cursos de Licenciatura em Matemática? Quais as principais orientações dos documentos oficiais, ao tratarem do ensino de função na Educação Básica? Quais os principais momentos da transposição didática, quando o conceito de função passa da comunidade científica para a comunidade escolar? Quais OM e OD são utilizadas no ensino de função nas licenciaturas em Matemática?

No caso da dimensão ecológica de um problema didático, existem questões relacionadas às restrições e condições praxeológicas diante dos níveis de codeterminação didática ${ }^{9}$. Os níveis de codeterminação didática (CHEVALLARD, 2001, 2002) permitem estudar e compreender as influências e restrições das $\mathrm{OM}$ e $\mathrm{OD}$, criando condições para reestruturar e reorganizar as condições de ensino e aprendizagem, a partir do nível mais genérico (civilização) ao mais específico (problema matemático). Gascón (2011, p. 219) resume a dimensão ecológica de um problema didático, ao afirmar que "envolve questões que pretendem indagar que tipo de restrições e de que nível provêm e são cruciais para a ecologia das praxeologias matemáticas e didáticas". Compreender as restrições e suas origens é essencial para criar condições ao desenvolvimento das praxeologias, isto é, à sua existência e razão de ser.

Portanto, temos as seguintes questões: $P_{3}(F)$ : Quais restrições dificultam $\mathrm{o}$ desenvolvimento da modelação funcional aplicado ao conceito de função e que condições são necessárias para superar essas dificuldades? Como construir e aplicar um PEP fundamentado no MER do conceito de função? Quais as condições necessárias para aplicar um PEP? Quais restrições podem existir no desenvolvimento de um PEP direcionado ao conceito de função? Quais os meios e recursos necessários para o desenvolvimento do PEP direcionado ao conceito de função?

Ao caracterizarmos as dimensões do problema didático, relacionado ao conceito de função, construímos um diagrama para o ensino do conceito de função que faz parte da estrutura do MER, no sentido de proporcionar respostas às dimensões, principalmente à dimensão ecológica.

\subsection{Construção do diagrama para o ensino do conceito de função}

A organização teórica para a construção do diagrama do conceito de função teve por

\footnotetext{
${ }^{9}$ Chevallard (2007) apresenta os níveis de codeterninação do mais alto que é a civilização e segue com sociedade, escola, pedagogia, disciplina, domínio, setor, tema e tópico que o mais baixo. Nesse sentido, a compreensão dos níveis de codeterminação busca identificar e compreender as restrições nos seus diferentes níveis, para proporcionar condições para o processo de ensino e aprendizagem de um determinado conceito (CHEVALLARD, 2002, 2007).
} 
base o caminho seguido por Lucas (2015), ao propor o diagrama de atividade de modelação funcional como esquema do MER. A proposta de Lucas (2015) é direcionada para o conceito do cálculo diferencial elementar, que utiliza a Modelação Matemática (CHEVALLARD, 1989; GASCÓN, 2001) e os níveis de Modelação Funcional (RUIZ-MUNZÓN, 2010). A seguir, apresentaremos a ideia de cada base teórica utilizada para a construção do diagrama.

Com a finalidade de organizar e representar as ideias da modelação matemática no âmbito da TAD, Chevallard (1989) apresenta um esquema simplificado do processo de modelação elementar, descrito em três etapas: a primeira define o sistema que queremos estudar, especificando os aspectos do sistema a que nos propomos estudar e o simbolizando mediante um conjunto de variáveis; a segunda constrói o modelo propriamente estabelecido, definindo as relações entre as variáveis identificadas na primeira etapa; a terceira utiliza o modelo definido com o objetivo de produzir conhecimento relativo ao sistema.

Tomando por base o esquema proposto por Chevallard (1989) e Gascón (2001), propõem-se quatro estágios para a modelação matemática, sem existir necessariamente uma ordem rigorosa a ser seguida: o primeiro delimita ou constrói o sistema de modelação em que formula questões problemáticas e conjecturas; o segundo constrói os modelos e formula as questões iniciais, envolvendo a definição ou a delimitação do sistema provisório elaborado anteriormente, elaborando o modelo matemático correspondente; o terceiro utiliza o modelo e interpreta, caracterizando um trabalho técnico dentro do modelo, analisa o trabalho e seus resultados no sistema modelizado. Esse estágio observa e decide a melhor aplicação e adequação do modelo e de suas técnicas utilizadas; e, por último, o quarto estágio representa um novo processo de modelação para novas questões, existindo a formulação de novos problemas, cujas resoluções responderão a novos questionamentos relativos ao sistema, dificilmente formulados antes da elaboração do modelo matemático, utilizando novas organizações matemáticas e novos modelos.

Os três níveis de modelação funcional de Ruiz-Munzón (2010) vêm como proposta para superar as dificuldades e restrições da vida escolar no processo de algebrização da Matemática. Os níveis estão relacionados à modelização de funções e seu desenvolvimento é progressivo: o primeiro nível aborda as funções isoladas de uma única variável, inclui as equações (ou inequações) associadas e as etapas de modelação algébrica propostas por Bolea (2002), em que se procura responder às questões que fazem referência à variação de uma variável em função da outra; o segundo nível é a família de funções de uma variável, que materializa os modelos que se expressam precisamente através das famílias de funções de uma variável e suas equações (ou inequações) paramétricas associadas, deixando claro a distinção entre parâmetros e 
variáveis; o terceiro nível aborda a família de funções de duas ou mais variáveis, que se expressa pelos modelos das famílias de funções de duas ou mais variáveis e suas correspondentes fórmulas associadas, tomando por base os modelos utilizados no segundo nível.

O trabalho de Lucas (2015) traz uma análise detalhada das condições institucionais necessárias para aplicar uma experimentação por meio do Percurso de Estudo e Pesquisa (PEP), para o ensino do cálculo diferencial elementar, em um curso de Medicina Nuclear do ensino universitário. O MER proposto por Lucas (2015) apresenta diversas características e funcionalidades que partem de questões epistemológicas do saber em jogo, até à análise da transposição didática em todo o processo. Procura identificar as restrições institucionais com a finalidade de construir condições para direcionar e fundamentar as propostas de ensino que possibilitem meios para o processo de aprendizagem do cálculo diferencial elementar.

Na sua reformulação da noção da modelização funcional e da ampliação dos níveis de Ruiz-Munzón, Lucas (2015) apresenta um esquema detalhado dos tipos de tarefas como componentes dos quatro estágios do processo de modelização matemática. O esquema do MER é apresentado por meio de um diagrama de atividades chamado de diagrama de atividade de modelização funcional. O diagrama apresenta os diferentes caminhos matemáticos a serem utilizados na resolução de uma questão, relacionando funções elementares e descrevendo um trabalho tanto no campo discreto como no campo contínuo. No desenvolvimento do estudo, outras questões podem surgir, integrando o processo de modelação e até reformulando as já existentes, manifestando um "caráter recursivo do processo de modelação matemática" (LUCAS, 2015, p. 93).

Com os elementos iniciais do MER proposto, juntamente com a organização teórica utilizada, propomos um esquema que procura diminuir as dificuldades no ensino da noção de função na Licenciatura em Matemática como outra alternativa de ensino, que apresentamos a seguir: 


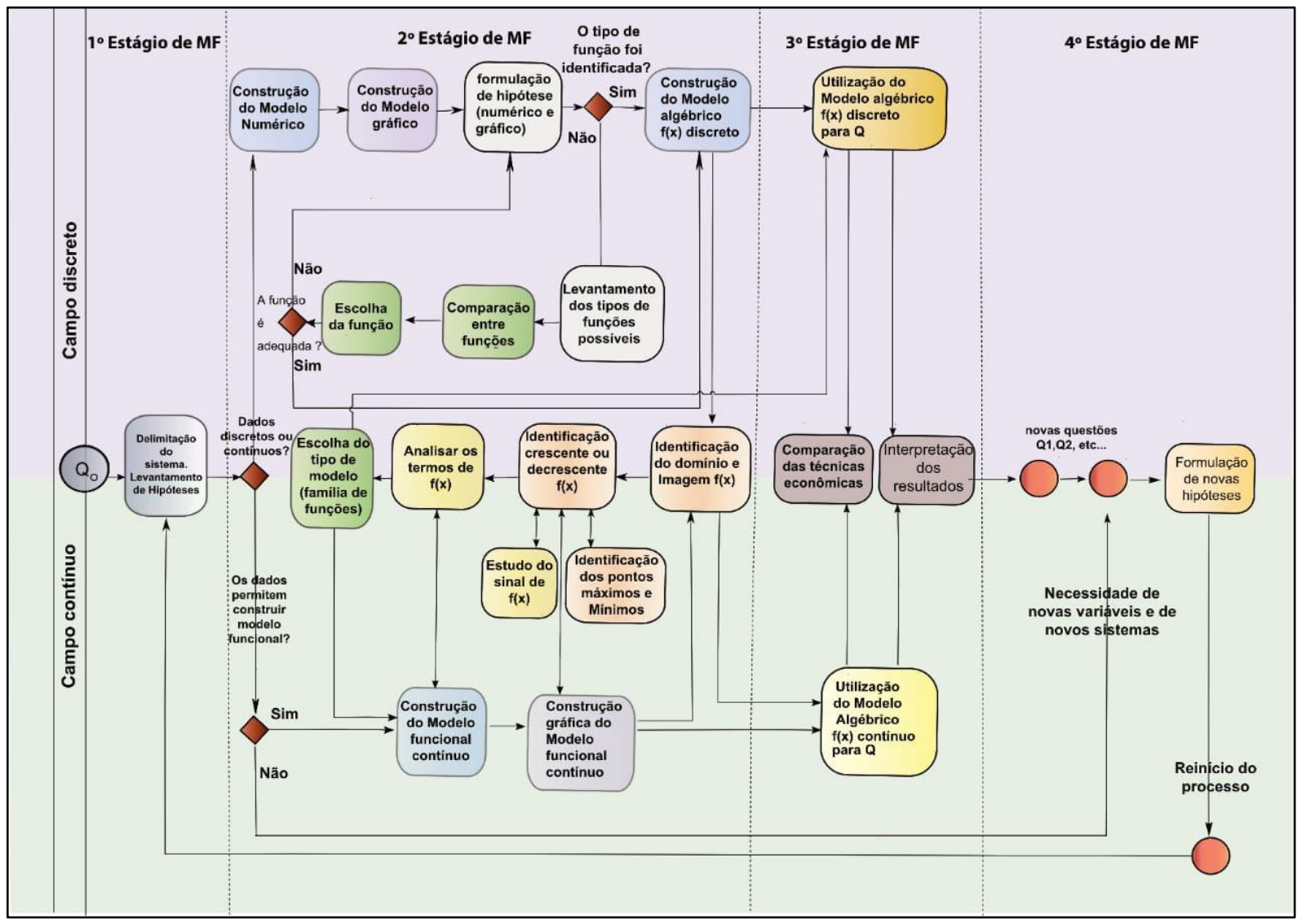

Figura 1 - Diagrama de atividade da noção de função Fonte: Rodrigues (2019, p. 156)

Este diagrama está organizado em dois principais campos: o contínuo e o discreto. A determinação inicial do tipo de dados a serem utilizados nos conduzirá pelos caminhos possíveis apresentados no diagrama. Os "retângulos" são as possíveis tarefas que os alunos deverão utilizar durante o processo e estão identificadas com as ações e agrupadas por cores como, por exemplo: as que possuem a cor azul estão ligadas às tarefas que têm como objetivo a construção de modelos. Os losangos representam decisões a serem tomadas pelos alunos no decorrer do processo; os círculos são questões desenvolvidas e suas derivadas. Destacamos que o diagrama representa um esquema provisório que pode ser alterado no decorrer do processo ou por sugestões apresentadas pela comunidade de professores ou pesquisadores.

O esquema está dividido em quatro estágios de modelação funcional: o primeiro está direcionado para o levantamento das informações preliminares para a construção do modelo funcional. A partir da questão geratriz, são levantadas as hipóteses e as possíveis questões derivadas $\left(Q_{1}, Q_{2}, Q_{3}, \ldots\right)$; no segundo estágio, existe a definição se os dados que possuem são contínuos ou discretos. Com-essa definição, o percurso de estudo e pesquisa percorre um possível caminho na resolução de tipos de tarefas com o propósito de construir e estudar o modelo funcional determinado no estudo; no terceiro estágio, temos a utilização do modelo e a 
interpretação dos resultados gerados, decidindo a melhor aplicação e adequação do modelo funcional determinado; por último, o quarto estágio, que é a elaboração de um novo processo de modelação para as novas questões.

\section{Descrição da aplicação do PEP do conceito de função tomando por base o MER proposto}

O material utilizado para a aplicação do PEP é composto pela ficha diagnóstica, ficha de trabalho e questão geratriz. Na montagem desse material, os percursos matemáticos $(\mathrm{PM})^{10}$ foram desenvolvidos tomando por base o MER proposto, com a finalidade de serem utilizados como referência na resolução da questão geratriz.

No caso das fichas, a sua elaboração foi fundamentada em Guidorizzi (2011), Iezzi e Murakami (2004); Lima, Carvalho, Wagner e Morgado (2004); Morettin, Hazzan e Bussab (2003); e Tinoco (2009). A ficha diagnóstica, com 8 itens, foi aplicada de maneira individual e apresentada à identificação gráfica de uma função, do valor numérico de sua imagem, a partir de uma função dada; da simplificação de expressão algébricas ou numéricas; da identificação do domínio e da imagem; da identificação se a função é crescente ou decrescente; da construção da representação algébrica e gráfica de uma função, a partir de dados discretos; da construção da representação algébrica e gráfica de uma função, a partir de dados contínuos; assim como de dados discretos para uma função contínua. A ficha de trabalho contém 10 (dez) itens que abordam a construção de gráficos no plano cartesiano, a análise de parâmetros observando o crescimento ou decrescimento, o zero da função, o estudo do sinal e a resolução de problemas. A utilização das fichas teve o propósito de revisar as técnicas mais usuais a serem utilizadas como ponto de partida na possível evolução das organizações matemáticas. No caso da questão geratriz, tinha o seguinte enunciado:

\section{Contextualização do Problema}

Aposentadoria é um tema que surge de tempos em tempos fazendo parte das discussões dos brasileiros, pois afeta diretamente a vida das pessoas economicamente ativas e de suas famílias. O Congresso Federal brasileiro aprovou a lei 13.183/2015 que trata da mudança de algumas regras para a aposentadoria, na qual um ponto em destaque trata da fórmula 85/95. No caso, a mulher terá que alcançar uma soma de 85 anos, somando tempo da sua contribuição com a sua idade. No caso do homem serão 95 anos, seguindo o mesmo princípio. As idades 85 e 95

\footnotetext{
${ }^{10}$ Os Percursos Matemáticos (PM) fundamentam as organizações matemáticas utilizadas para a resolução da questão geratriz, na busca de construir uma resposta adequada ao problema. Os PM propõem um aumento gradual do uso dos tipos de praxeologias, levando o aluno a utilizar técnicas conhecidas e mobilizando duas ou mais delas na busca da resposta.
} 
anos levam em conta a expectativa de vida dos brasileiros até 2018 .

Para os anos seguintes, teremos:

$$
\begin{aligned}
& 2019 \text { a 2020: } 86 \text { (mulheres) / } 96 \text { (homens); } \\
& 2021 \text { a 2022: } 87 \text { (mulheres) / } 97 \text { (homens); } \\
& \text { 2023 a 2024: } 88 \text { (mulheres) / } 98 \text { (homens); } \\
& \text { 2025 a 2026: } 89 \text { (mulheres) / } 99 \text { (homens); } \\
& \text { 2027: } 90 \text { (mulheres) / } 100 \text { (homens). } \\
& \text { Fonte: Agência Senado (CASTRO; VILAR, 2015) }
\end{aligned}
$$

$\mathrm{Q}_{0}$ : Como representar a situação atual e futura dos casos de aposentadorias das mulheres e dos homens, tomando por base a Lei 13.183/2015, que trata das mudanças de algumas regras para as aposentadorias?

A aplicação do PEP foi dividida em duas etapas, compostas de 6 (seis) seções presenciais (SP) e de sessões não presenciais (SNP). Para a sua aplicação, seguimos um cronograma proposto separado em sessões presenciais (SP), que consta da atividade diagnóstica sobre o conceito de função, da ficha de trabalho que aborda o conceito de função afim, da questão geratriz e de suas derivadas. As seções não presenciais (SNP) aconteceram em horários diferentes das aulas da disciplina Funções I. Nesses momentos os alunos utilizaram os recursos tecnológicos para a realização de pesquisas, a construção dos gráficos com o auxílio do GeoGebra e a utilização de cálculos longos.

\subsection{Local, sujeitos e instrumentos de coletas da pesquisa}

Os sujeitos da pesquisa foram o professor e seus alunos da disciplina Funções I, da Licenciatura em Educação do Campo com habilitação em Matemática. A disciplina faz parte da Matriz Curricular do curso e apresenta, em sua ementa, o conteúdo de função.

Para a coleta de dados, utilizamos filmagens, gravações de áudio, protocolos e o caderno de anotações. Para as filmagens, utilizamos duas câmeras posicionadas em ângulos diferentes. Para a captação dos áudios, foram utilizados três gravadores colocados em locais diferentes da sala de aula. Os protocolos relacionados a resoluções individuais e coletivas das questões foram coletados e analisados. Estimulou-se os alunos a deixarem registrados todos os procedimentos o mais detalhadamente possível, para identificarmos a praxeologia inicialmente utilizada, e suas mudanças. Em relação ao caderno de anotações, nele registramos todos os momentos relevantes da pesquisa, principalmente a descrição de acontecimentos que a filmagem e a gravação de áudio não deixaram claros, relacionando momentos das aulas aos pontos das teorias utilizadas na pesquisa. 


\section{Análise dos encontros presenciais}

A aplicação e análise do MER foram iniciadas a partir da terceira seção presencial, tendo em vista que os dois primeiros encontros foram direcionados à aplicação das fichas diagnóstica e de trabalho, que tinham a função de relembrar e consolidar as técnicas usuais. Com a aplicação da questão geratriz, iniciamos a aplicação e verificação da conformidade com o MER.

Ao aplicarmos a questão geratriz no terceiro encontro, tomando por base o MER, iniciamos a análise do desenvolvimento do modelo proposto. Primeiramente foi identificada a realização do primeiro estágio da modelação funcional, que é o contato com a questão geratriz e o levantamento de hipóteses, gerando questões derivadas a partir da questão geratriz. $\mathrm{Na}$ ocasião, o professor apresentou a questão geratriz e, a partir dela, levantou alguns questionamentos juntamente com os alunos, como por exemplo: idade inicial para começar a contribuir; a relação idade e tempo de contribuição; idade máxima para aposentadoria; e a diferença na aposentadoria entre homens e mulheres. Na continuidade do terceiro encontro, os alunos organizaram os dados levantados, utilizando quadros com valores discretos da idade e do tempo de contribuição para homens e mulheres, tomando por base uma pessoa que começou a contribuir aos 18 anos de idade. Um dos problemas iniciais percebidos no desenvolvimento do PEP foi a intervenção efetiva do professor na elaboração das questões e na organização dos dados, restringindo o espaço para a participação dos alunos. Consequentemente, com a elaboração do modelo numérico pelos alunos ${ }^{11}$, iniciamos o segundo estágio de modelação funcional proposto pelo MER.

No quarto encontro, os alunos determinaram as funções do tempo de contribuição (TC) ${ }^{12}$ para mulheres e para homens. Como os alunos foram organizados em grupos, passaram a utilizar o GeoGebra com a finalidade de observar as representações gráficas de cada função, determinando o domínio e a sua imagem com o auxílio do professor. Ao final do encontro, verificamos uma autonomia maior por parte dos alunos, na medida que a participação na busca da resposta da questão geratriz é compartilhada. Também observamos uma mudança na condução praxeológica do professor, a partir da sua abordagem durante a aplicação do PEP, em que vários questionamentos são levantados por ele, com a finalidade de levar os alunos a refletirem sobre suas respostas, tornando presente a dialética pergunta e resposta proposta pelo

\footnotetext{
11 Os alunos utilizaram os dados relacionados a questão, como: idade inicial de contribuição; e tempo de contribuição. Com os dados, buscaram relacionar e organizar as informações utilizando um quadro com valores numéricos, com a finalidade de buscar a generalização.

${ }^{12} T C=\frac{85-I}{2}$, função Tempo de Contribuição para mulheres. No caso dos homens foi utilizado o valor de 95 no lugar de 85 . A variável $I$, representa a idade inicial de contribuição.
} 
PEP (FONSECA; CASAS; BOSCH; GASCÓN, 2009).

No encontro seguinte, com a função tempo de contribuição definida, juntamente com o seu domínio e a sua imagem, os alunos iniciaram o estudo de outros elementos pertencentes às funções. Na imagem seguinte, podemos observar o percurso de estudo realizado pelos alunos com o auxílio do professor.

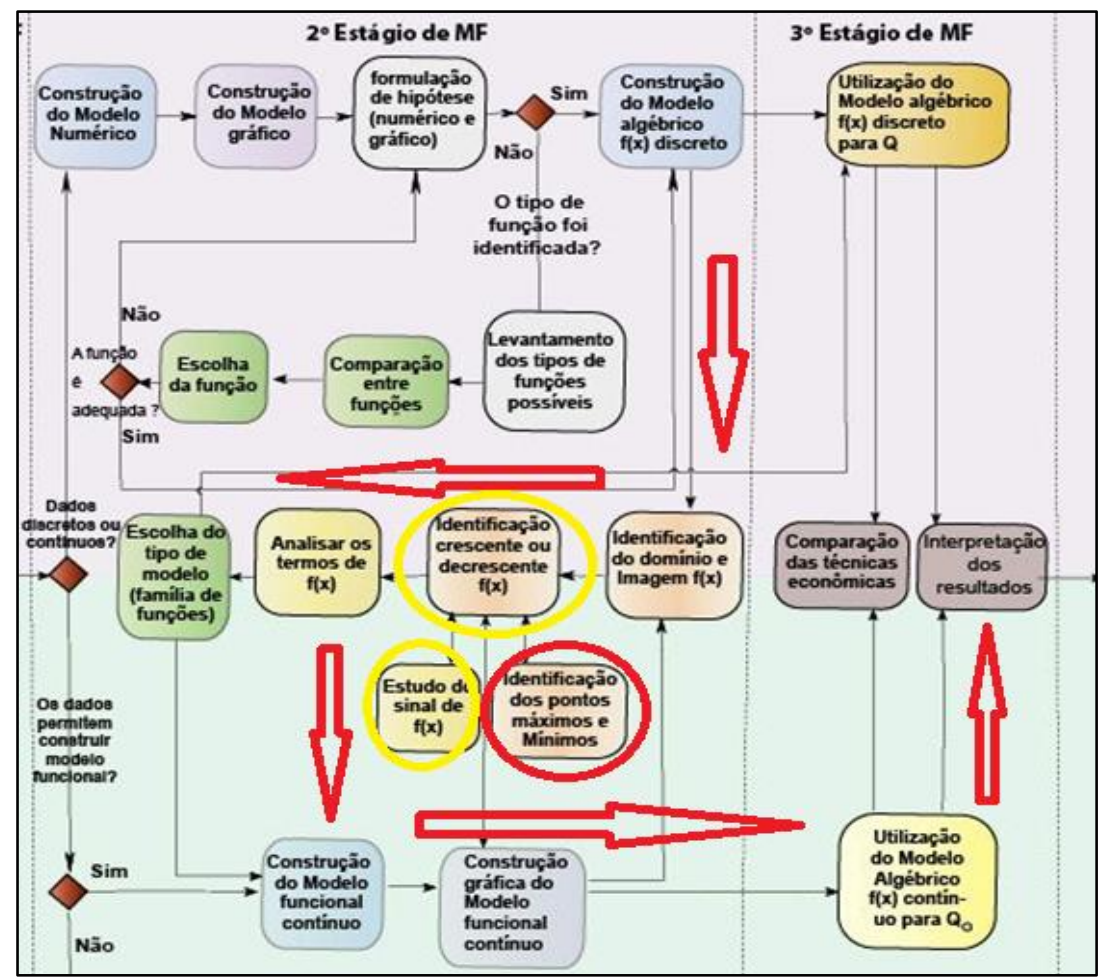

Figura 2 - Parte do MER, representando o caminho seguido no quinto encontro Fonte: Rodrigues (2019, p. 258)

Observamos que analisaram os termos da função tempo de contribuição, construíram o modelo funcional contínuo e o seu gráfico, em seguida, utilizaram o modelo funcional para interpretar os resultados. Destacamos que o professor não realizou uma discussão relacionada ao ângulo de inclinação da reta, destacada em amarelo, o que permitiria discutir se a função é crescente ou decrescente, ponto importante ao se tratar de um estudo da função afim. Mas, ao abordar sobre os valores do domínio e da imagem, o professor identificou os seus pontos máximos e mínimos, adequando a sua representação ao contexto do problema.

Identificamos um caminho realizado pelos alunos não previsto no esquema inicial, no caso, foi a passagem da tarefa de análise dos termos de uma função para a construção do modelo funcional contínuo. Esse fato é aceito em qualquer MER, pois o modelo é flexível e cada componente dele pode ser mudado ou adaptado (BOSCH; GASCÓN, 2010). Com a construção do gráfico funcional contínuo do tempo de contribuição $(T C)$, o processo é repetido para a 
construção do gráfico funcional contínuo de anos complementares $(A C)^{13}$, concluindo o segundo estágio do modelo funcional do MER. Em seguida, os alunos utilizaram os modelos algébricos $T C$ e $A C$, consequentemente seguindo para a interpretação dos resultados e finalizando o terceiro estágio do modelo funcional.

Analisando, a partir das seções presenciais previstas, esse encontro abordou as questões derivadas ${ }^{14} \mathrm{Q}_{1,3,2}$ e $\mathrm{Q}_{1,3,3}$, no caso, inicialmente para as mulheres. Porém outras questões foram introduzidas pelo professor durante o desenvolvimento do PEP, no caso, as questões derivadas: $\mathrm{Q}_{1,3,3,1}$ : Como podemos representar graficamente os Anos Complementares (AC)?; $\mathrm{Q}_{1,3,3,2}$ : Qual o domínio e imagem de AC?. Essas questões tratam sobre a determinação do domínio e imagem de $A C$ e sua construção gráfica, questões que não estavam previstas antes da aplicação, referendando que a aplicação de um PEP não é totalmente pré-definida, existindo espaço para novos questionamentos (CHEVALLARD, 2009a, 2009b).

No último encontro presencial do estudo, foi desenvolvida a análise das famílias de funções $\left(\mathrm{Q}_{2}\right)^{15}$. Os alunos construíram as famílias de funções, utilizando o GeoGebra, assim como discutiram o significado e aplicação das representações gráficas em situações possíveis, ao tratar da aposentaria. $\mathrm{Na}$ imagem seguinte, apresentamos o percurso de estudo e pesquisa realizado pelos alunos, ao tentar resolver a $\mathrm{Q}_{2}$ :

\footnotetext{
${ }^{13} A C=30-T C$, função Anos Complementares para mulheres e homens, foi considerando o valor mínimo de contribuição de 30 anos.

${ }^{14} \mathrm{Q}_{1,3,2}$ :Com a função TC definida, qual o seu domínio e a sua Imagem? e $\mathrm{Q}_{1,3,3}$ : Como podemos representar graficamente o tempo de contribuição se a mulher começar depois dos 25 anos?

${ }^{15} \mathrm{Q}_{2}$ : Como podemos representar as situações para os anos seguintes, sabendo que a soma idade mais tempo de contribuição muda a cada dois anos?
} 


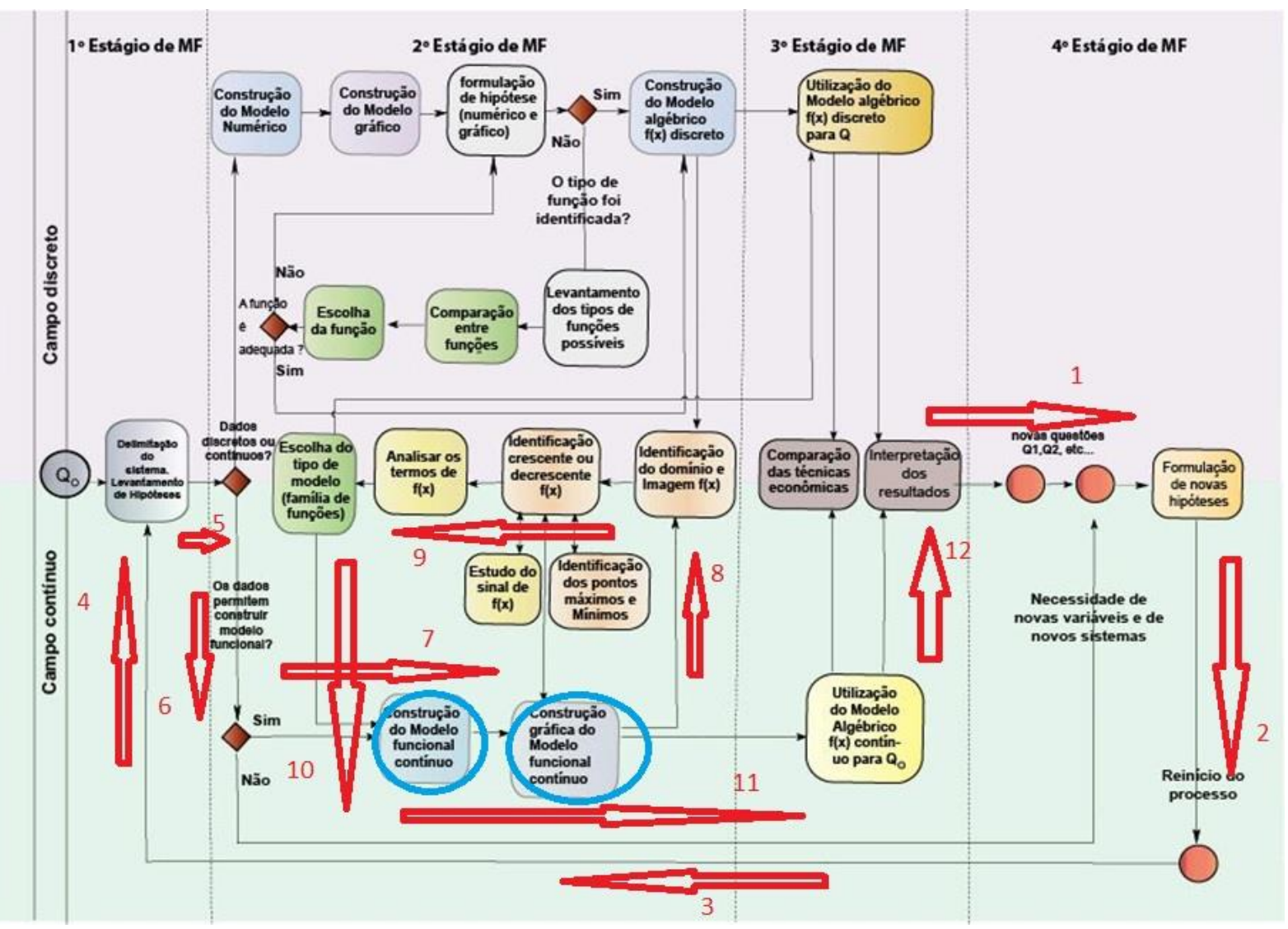

Figura 3 - Percurso dos grupos durante o sexto encontro Fonte: Rodrigues (2019, p. 266)

Os alunos, ao se depararem com novas questões geradas pela $\mathrm{Q}_{0}$, representadas pelas setas de 1 a 5, formulam novas hipóteses e tentam delimitar o caminho a ser seguido. Como já possuem o modelo funcional e contínuo, partem para a identificação do domínio e imagem, setas de 6 a 8, em seguida, fazem o estudo do sinal, analisam os termos da função e o modelo da família de funções para as construções gráficas, setas de 7 a 11 e, consequentemente, a interpretação dos resultados, seta 12. Os tipos de tarefas que estão marcadas por azul na imagem anterior, são as etapas que não foram efetivadas pelos alunos, pelo fato de não ser necessária a construção do modelo funcional. O registro no GeoGebra das limitações dos domínios e imagens não foram realizados por falta de tempo.

No decorrer dos seis encontros presenciais, os tipos de tarefas propostas pelo PEP levaram os alunos a utilizarem técnicas já conhecidas, tais como: a determinação do zero da função por meio das etapas para resolver uma equação do primeiro grau; o método da adição para a resolução de um sistema de duas variáveis e o método da construção de gráficos de uma função do primeiro grau, utilizando dois valores diferentes do domínio. Como também, aperfeiçoaram técnicas para determinar as funções de $T C$ e $A C$, apoiados por um amparo 
tecnológico. Nesta aplicação, podemos perceber o crescimento das organizações matemáticas ${ }^{16}$ utilizadas pelos alunos que partiram de organizações matemáticas pontuais, passaram para organizações matemáticas locais e chegaram a uma compreensão da organização matemática regional (CHEVALLARD, 1999).

\section{Conclusão}

Ao analisarmos o MER proposto, observamos que ele sofreu poucas alterações durante a aplicação do dispositivo didático. Foram abordadas a construção, interpretação e aplicação de funções discretas, como também a transição de um domínio discreto para um domínio contínuo, buscando a compreensão de uma função contínua. Também foi abordado o estudo dos termos de uma função, famílias de funções, assim como a sua variabilidade. Uma questão que devemos ressaltar é a compreensão da relação entre as grandezas de uma função, um conceito básico e que, muitas vezes, as técnicas tomam um papel de destaque, ofuscando os conceitos essenciais para compreensão da funcionalidade e aplicação do conceito.

$\mathrm{Na}$ aplicação do dispositivo didático, buscamos seguir o planejamento inicial apresentado no cronograma proposto. O professor teve toda liberdade de alterar e propor mudanças que achasse necessário. Nesse sentido, fez as alterações e seguiu sua organização didática, tomando por base a proposta metodológica. Com esse procedimento, o professor sentiu-se à vontade para conduzir as sessões, em que surgiram várias situações didáticas não esperadas, a partir de perguntas realizadas pelos alunos que foram além do planejamento.

Tal fato é condizente com a proposta do PEP, um desenvolvimento sem as amarras de um planejamento rígido, mas com objetivos para buscar a compreensão do conceito abordado e de sua aplicabilidade (CHEVALLARD, 2009a, 2009b). Consequentemente, as mudanças praxeológicas apresentadas pelo professor durante a aplicação do dispositivo didático, caracterizam uma influência positiva em sua formação.

Ressaltamos que o trabalho com a proposta do MER foi realizada no curso de licenciatura em Matemática para a Educação do Campo, que possui características específicas em sua estrutura, na sua condução metodológica e na sua formação. Ademais, a abordagem sobre o conceito de função no estudo não explora toda a sua amplitude epistemológica, e nem

\footnotetext{
${ }^{16}$ O PEP é aplicado e desenvolvido tomando por base a complexidade crescente das organizações matemáticas, isto é, a partir de uma organização matemática pontual (OMP), espera chegar a uma organização matemática local (OML), e, consequentemente a uma organização matemática regional (OMR) e a uma organização matemática global (OMG).
} 
todos os tipos de funções elementares. Por esses fatos, temos a compreensão que o MER proposto deve ser aplicado em outros tipos de licenciaturas, assim como a continuidade dos estudos relacionados com o conceito de função, com a intenção de complementarmos a proposta continuamente.

\section{Agradecimentos}

O presente trabalho foi realizado com apoio da Coordenação de Aperfeiçoamento de Pessoal de Nível Superior - Brasil (CAPES) - Código de Financiamento 001. Agradecemos também, o apoio da Faculdade de Psicologia e de Ciências da Educação da Universidade do Porto e do Instituto Federal do Rio Grande do Norte (Campus Canguaretama).

\section{Referências}

ALMOULOUD, S. A.; SILVA, M. J. F. Engenharia didática: evolução e diversidade. Revemat, Florianópolis, v. 7, n. 2, p. 22-52, 2012. Disponível em: https://periodicos.ufsc.br/index.php/revemat/article/view/1981-1322.2012v7n2p22. Acesso em: 25 jun. 2019.

ARTIGUE, M. Engenharia didática. In: BRUN, J. (Org.). Didáctica das matemáticas. Lisboa: Instituto Piaget, 1996. p. 193-217.

BOLEA, P. El proceso de algebrización de organizaciones matemáticas escolares. Tesis (Doctorado en Matemáticas) - Universidad de Zaragoza, Zaragoza, 2002.

BOSCH, M.; GASCÓN, J. Fundamentación antropológica de las organizaciones didáticas: de los "talleres de prácticas matemáticas" a los "recorridos de estudio e investigación". In: BRONNER, A. (Org.). Diffuser les mathématiques (et les autres savoirs) comme outils de connaissance et d'action. Montpellier: Université de Montpellier, 2010. p. 55-91.

BOYER, C. B. História da Matemática. São Paulo: Edgard Blücher, 1996.

BRASIL. Ministério da Educação e Desporto. Parâmetros curriculares nacionais: terceiro e quarto ciclos do ensino fundamental. Brasília: Ministério da Educação e Desporto, 1998. 175 p. Disponível em: http://portal.mec.gov.br/seb/arquivos/pdf/introducao.pdf. Acesso em: 26 jun. 2019.

BRASIL. Ministério da Educação. Parâmetros curriculares nacionais (ensino médio): ciências da natureza, matemática e suas tecnologias. Brasília: Ministério da Educação, 2000. 58 p. Disponível em: http://portal.mec.gov.br/seb/arquivos/pdf/ciencian.pdf. Acesso em: 26 jun. 2019.

BRASIL. Ministério da Educação. Orientações curriculares para o ensino médio: Ciências da Natureza, Matemática e suas tecnologias. Brasília: Ministério da Educação, 2006. 140 p. 2 v. Disponível em: http://portal.mec.gov.br/seb/arquivos/pdf/book_volume_02_internet.pdf. Acesso em: 26 jun. 2019.

BRASIL. Ministério da Educação. Base nacional comum curricular. Brasília: Ministério da Educação, 2017. 600 p. Disponível em:

http://basenacionalcomum.mec.gov.br/images/BNCC_EI_EF_110518_versaofinal_site.pdf. Acesso 
em: 26 jun. 2019.

CASTRO, A.; VILLAR, I. Senado estabelece novas regras para a aposentadoria. Senado Notícias. 2015. Disponível em: https://www12.senado.leg.br/noticias/materias/2015/10/07/senado-aprova-mpque-estabelece-novas-regras-para-aposentadoria. Acesso em: 30 nov. 2016.

CHEVALLARD, Y. Arithmétique, algèbre, modélisation: étapes d'une recherche. Marselha: AixMarseille, 1989.

CHEVALLARD, Y. Conceitos fundamentais da didática: as perspectivas trazidas por uma abordagem antropologica. In: BRUN, J. (Org.). Didáctica das matemáticas. Lisboa: Instituto Piaget, 1996. p. $115-153$.

CHEVALLARD, Y. L'analysedes pratiques enseignantesen theorie anthropologie didactique. In: RECHERCHES EN DIDACTIQUES DES MATHEMATIQUES, 6., 1999, Marselha. Anais [...]. Marselha: Aix-Marseille, 1999. p. 1-29. Disponível em:

http://yves.chevallard.free.fr/spip/spip/IMG/pdf/Analyse_des_pratiques_enseignantes.pdf. Acesso em: 27 jun. 2019.

CHEVALLARD. Y.; BOSCH, M.; GASCÓN, J. Estudar matemáticas: o elo perdido entre o ensino a aprendizagem. Porto Alegre: Artes Médicas, 2001.

CHEVALLARD, Y. Organiser l'étude 1. Structures et fonctions. In: ÉCOLE D'ÉTÉ DE DIDACTIQUE DES MATHÉMATIQUES, 11., 2002, Grenoble. Anais [...]. Grenoble: Université Grenoble, 2002. p. 03-32. Disponível em:

http://yves.chevallard.free.fr/spip/spip/IMG/pdf/Organiser_1_etude_1.pdf. Acesso em: 27 jun. 2019.

CHEVALLARD, Y. Vers une didactique de lacodisciplinarité. 2004. Disponível em:

http://yves.chevallard.free.fr/spip/spip/IMG/pdf/Vers_une_didactique_de_la_codisciplinarite.pdf. Acesso em: 26 jun. 2019.

CHEVALLARD, Y. Steps towards a new epistemology in mathematics education. In: CONGRESS OF THE EUROPEAN SOCIETY FOR RESEARCH IN MATHEMATICS EDUCATION, 4., 2006, Barcelona. Anais [...]. Barcelona: FUNDEMI-IQS, 2006. p. 21-30. Disponível em: http://yves.chevallard.free.fr/spip/spip/IMG/pdf/Steps_towards_a_New_Epistemology.pdf. Acesso em: 26 jun. 2019.

CHEVALLARD, Y. Passé et présent de la théorie anthropologique du didactique. In: RUIZHIGUERAS, L.; ESTEPA, A.; GARCÍA, F. J. (Orgs.). Sociedad, escuela y matemáticas: aportaciones de la teoría antropológica de la didáctica. Jaén: Servicio de publicaciones de la Universidad de Jaén, 2007. p. 705-746.

CHEVALLARD, Y. La notion de PER : problèmes et avancées. 2009a. Disponível em: http://yves.chevallard.free.fr/spip/spip/IMG/pdf/La_notion_de_PER__problemes_et_avancees.pdf. Acesso em: 27 jun. 2019.

CHEVALLARD, Y. Remarques sur la notion d'infrastructure didactique et sur le rôle des PER. In: APPRENTISSAGE, DIDACTIQUE, ÉVALUATION, FORMATION, 1., 2009, Lyon. Anais [...]. Lyon: UMR, 2009b. p. 1-31. Disponível em:

http://yves.chevallard.free.fr/spip/spip/article.php3?id_article=155. Acesso em: 27 jun. 2019.

D’AMORE, B. Elementos de didática da matemática. São Paulo: Livraria da Física, 2007.

ESPITIA, L. E. S.; CRUZ, K. J. C.; OCHOA, C. P. F. Influencias del contrato didáctico en el aprendizaje del concepto de función. Praxis e Saber: Revista de Investigación y Pedagogía, [s. l.], v. 
3, n 3. p. 119-138, 2011

EVES, H. Introdução à história da matemática. Campinas: Editora Unicamp, 2004.

FONSECA, C. et al. Diseño de un recorrido de estudio e investigación en los problemas de modelización. In: SIMPOSIO DE LA SOCIEDAD ESPAÑOLA DE INVESTIGACIÓN EN EDUCACIÓN MATEMÁTICA, 13., 2009, Santander. Anais [...]. Santander: Universidad de Cantabria, 2009. p. 1-7. Disponível em:

http://www.seiem.es/docs/comunicaciones/GruposXIII/dmdc/Fonseca_Casas_Bosch_Gascon_R.pdf. Acesso em: 29 jun. 2019.

GASCÓN, J. Incidencia del modelo epistemológico de las matemáticas sobre las prácticas docentes.

Revista Latinoamericana de Investigación en Matemática Educativa, Ciudad de Mexico, v. 4, n. 2, p. 129-159, 2001. Disponível em: http://www.redalyc.org/pdf/335/33540202.pdf. Acesso em: 29 jun. 2019.

GASCÓN, J. Las tres dimensiones fundamentales de un problema didáctico: el caso del álgebra elemental. Revista Latinoamericana de Investigación en Matemática Educativa, Ciudad de Mexico, v. 14, n. 2, p. 203-231, feb. 2011. Disponível em:

http://www.scielo.org.mx/pdf/relime/v14n2/v14n2a4.pdf. Acesso em: 29 jun. 2019.

GASCÓN, J. Los modelos epistemológicos de referencia como instrumentos de emancipación de la didáctica y la historia de las matemáticas. Educación Matemática, Ciudad de Mexico, v. 336, p. 99 123, mar. 2014. Disponível em: http://www.redalyc.org/pdf/405/40540854006.pdf. Acesso em: 29 jun. 2019.

GUIDORIZZI, H. L. Um curso de cálculo. 5. ed. Rio de Janeiro: LTC, 2011.

IEZZI, G.; MURAKAMI, C. Fundamentos de matemática elementar 1: conjuntos, funções. 8. ed. São Paulo: Atual, 2004.

LIMA, E. L. et al. A matemática do ensino médio. 7. ed. Rio de Janeiro: Sociedade Brasileira de Matemática, 2004.

LIMA, R. A. Dificuldades dos alunos no estudo da função afim. 2014. Dissertação (Mestrado em Matemática) - Universidade Regional de Blumenau, Blumenau, 2014.

LORENÇO, E. H.; OLIVEIRA, P. C. O conceito de função na produção acadêmica da PUC/SP via registros de representação semiótica. Educação Matemática Pesquisa, Consolação, v. 16, n. 2. p. 369-383, 2014. Disponível em: https://revistas.pucsp.br/index.php/emp/article/view/17380. Acesso em: 29 jun. 2019.

LUCAS, C. O. Una posible "razão de ser" del cálculo diferencial elemental en el ámbito de la modelización funcional. 2015. Tese (Doutorado em Matemática) - Universidade de Vigo, Vigo, 2015.

MINISTÉRIO DA EDUCAÇÃO E CULTURA (MEC). Ofertas de Cursos de Licenciatura em Educação do Campo - Procampo. Disponível em: http://portal.mec.gov.br/pnaes/194-secretarias112877938/secad-educacao-continuada-223369541/18784-oferta-de-cursos-de-licenciatura-emeducacao-do-campo-procampo. Acesso em: 29 jun. 2019.

MORETTIN, P. A.; HAZZAN, S.; BUSSAB, W. Cálculo: funções de uma e várias variáveis. São Paulo: Saraiva, 2003.

NEVES, J. D.; RESENDE, M. R. O processo de ensino-aprendizagem do conceito de função: um estudo na perspectiva da teoria histórico-cultural. Educação Matemática Pesquisa, Consolação, v. 
18, n. 2, p. 599-625, 2016. Disponível em: https://revistas.pucsp.br/emp/article/view/23866. Acesso em: 29 jun. 2019.

ONUCHIC, L. R.; ALLEVATO, N. S. G. Formação de Professores: mudanças urgentes na licenciatura em matemática. In: FROTA, M. C. R.; NASSER, L. (Orgs.). Educação matemática no ensino superior: pesquisas e debates. Recife: SBEM, 2009. p. 169-187.

PARRA, V. E.; OTERO, M. R. Praxeologias didácticas en la universidad y el fenômeno del $<$ encierros>: un estudio de caso relativo al limite y continuidad de funciones. In: BOSCH, M. et al. (Orgs.). Aportaciones de la teoría antropológica de lo didáctico: un panorama de la TAD. Barcelona: Centre de Recerca Matemática, 2011. p. 719-740.

RODRIGUES, R. F.; MENEZES, M. B.; CÂMARA DOS SANTOS, M. Licenciatura em matemática e o percurso de estudo e pesquisa: uma proposta do modelo epistemológico de referência para o ensino e aprendizagem do conceito de função. Amazônia, Belém, v. 14, n. 27, p. 36 - 50, 2017.

RUIZ-MUNZÓN, N. La introducción del álgebra elemental y su desarrollo hacia la modelización funcional. 2010. Tese (Doutorado em Matemática) - Universitat Autònoma de Barcelona, Barcelona, 2010. Disponível em: https://dialnet.unirioja.es/servlet/tesis?codigo=22189. Acesso em: 30 jun. 2019.

TENÓRIO, A.; PENNA, P.; TENÓRIO, T. O uso de jogos da plataforma Mangahigh no estudo de funções polinomiais do $1^{\circ}$ grau. Educação Matemática Pesquisa, São Paulo, v. 17, n. 2. p. 257-280, 2015. Disponível em: https://revistas.pucsp.br/emp/article/view/21966. Acesso em: 30 jun. 2019.

TINOCO, L. A. A. (Org.). Construindo o conceito de função. Rio de Janeiro: Instituto de Matemática, 2009.

Submetido em 18 de Março de 2020. Aprovado em 19 de Novembro de 2020. 